\title{
AESTHETIC EVALUATION OF PLANT LANDSCAPE BASED ON PRINCIPAL FACTOR ANALYSIS AND SBE IN WETLAND PARK - A CASE STUDY OF JINLONG LAKE WETLAND PARK (CHINA)
}

\author{
Xuehong $\mathrm{TAN}^{1^{*}}$, Xinghui $\mathrm{LI}^{2}$, Yunle PENG ${ }^{3}$ \\ ${ }^{1}$ School of Environmental Engineering, Xuzhou University of Technology, 221008 Xuzhou, China \\ ${ }^{2}$ Xuzhou Geruilin Landscape Design Co., Ltd, 221008 Xuzhou, China \\ ${ }^{3}$ School of Arts, Xuzhou University of Technology, 221008 Xuzhou, China
}

Received 18 September 2020; accepted 16 December 2020

\begin{abstract}
Highlights
- The SBE value fluctuated greatly and the evaluation value of the principal factor analysis did not.

- The principal factor analysis method was suitable for the overall planning and design.

- The SBE method was more suitable for the creation of special landscape in the detailed design.

- The plant landscape design of wetland park should emphasize the application of hygrophyte and aquatic plant.

Abstract. The aesthetic value of 35 plant landscape samples of Jinlong lake wetland park in Xuzhou city of China were evaluated with principal factor analysis and SBE method. The results showed that: The SBE value of 35 plant landscape samples fluctuated greatly and the evaluation value of the principal factor did not change much. The order of SBE mean values of different types was: plant landscape on both sides of the roads $>$ plant landscape beside buildings and surroundings > typical plant community $>$ waterfront plant landscape. The order of value of the principal factor analysis of different types was: plant landscape on both sides of the roads $>$ typical plant community $>$ plant landscape beside buildings and surroundings $>$ waterfront plant landscape. The principal factor evaluation method contained more than $90 \%$ information of seven evaluation factors, while the SBE method mainly evaluated from "plant aesthetic factors". The satisfaction of waterfront plant landscape was low, the transformation of plant landscape should focus on increasing the species of waterfront plants, especially native plants, building natural revetments, creating rich canopy lines.
\end{abstract}

Keywords: plant landscape, SBE (Scenic beauty evaluation), wetland park, principal factor analysis, Xuzhou.

\section{Introduction}

Urban wetland park not only have ecological benefits, but also have high ornamental value and scientific value ( $\mathrm{Li}$ et al., 2012; Lin et al., 2013; Tan \& Chen, 2017). Although the construction of urban wetland park had entered a period of rapid development, there were still many problems in the construction of wetland park, such as excessive artificial intervention, not paying attention to the protection of animals and plants, large-scale development of entertainment projects and so on (Zhang et al., 2013; Xu et al., 2013). At present, there were many researches on urban wetland park, but they mainly focused on ecological benefits and landscape layout, and less on landscape aesthetics (Fan et al., 2018; Chen et al., 2018; Li et al., 2015;
Wang et al., 2015; Tan et al., 2020). The aesthetic value of landscape had a certain impact on the ecological sustainability of the landscape. If the landscape was beautiful, the landscape was easier to be protected, and if the landscape was ugly, the landscape was easy to be destroyed (Zhou et al., 2012; Gobster et al., 2007). Therefore, the research on the landscape aesthetic value of urban wetland park was of great significance to give full play to the maximum value of wetland park.

Plants were the most vital and representative elements in the wetland park, which could best reflect local characteristics (An et al., 2014). Besides water area, green space was the largest landscape element in urban wetland park, so aesthetic value of plant landscape directly affected the aesthetic value of the whole wetland

*Corresponding author. E-mail: snowman100@126.com 
park. With the development of landscape aesthetic evaluation, some landscape aesthetics evaluation studies on urban park, residential green space, forest landscape, rural landscape, wetland park and so on (Xu et al., 2017; Duan et al., 2018; Zhou et al., 2006; Chen et al., 2014; Yao \& Wu, 2017; Tan \& Peng, 2020; Ji et al., 2016; Kerebel et al., 2019). The research of some scholars developed from the ecological community protection to the artistic conception of wetland park, which layed an aesthetic foundation for the plant landscape construction of urban wetland park (Valdez et al., 2019). The main methods used were scenic beauty evaluation (SBE), analytic hierarchy process (AHP), semantic differential (SD), etc. Some comparative studies had been done. But as the most commonly used method of comprehensive evaluation, principal factor analysis was rarely used in landscape aesthetic evaluation. Principal factor analysis was widely used in environmental, economic, social and other fields because of its simplicity and objectivity. Aesthetic of plant landscape in Jinlong lake wetland park was evaluated with principal factor analysis method and the SBE method in this paper. On one hand, this study could provide certain guidance for the plant landscape design of urban wetland park, on the other hand, it could explore the applicability of the two methods. This study could also provide a theoretical basis for the construction of scenic and tourist like urban wetland park landscape.

\section{Study area overview}

Xuzhou was located in the southeast of the North China plain and at the junction of Jiangsu, Shandong, Henan and Anhui provinces. It was between $116^{\circ} 22^{\prime}-118^{\circ} 40^{\prime} \mathrm{E}$ and $33^{\circ} 43^{\prime}-34^{\circ} 58^{\prime}$ N. By 2019 , Xuzhou covered an area of 11,258 square kilometers and had a permanent resident population of $8.825,600$. Xuzhou was a part of the Yellow Sea plain and had a warm temperate monsoon climate with an average annual rainfall of $847.9 \mathrm{~mm}$ and the zonal vegetation was deciduous broad-leaved forest. There were crisscross rivers, lakes and reservoirs in Xuzhou city (Tan \& Peng, 2019). Jinlong lake wetland park was located in the southeast of Xuzhou city, it was a comprehensive scenic spot integrating ecology, tourism, leisure and business, mainly around the large artificial lake of Jinlong lake. Jinlong lake wetland park was the core content of Xuzhou high -speed rail international business district and the lake surface and landscape area were about 65 hectares. Jinlong Lake wetland park was planned and designed by Eastwood company of the United States. The water area of Jinlong lake was about 25 hectares and the average water depth was $4.5 \mathrm{~m}$. The landscape area was 40 hectares, divided into 4 districts with spring, summer, autumn and winter, 12 gardens, 1 island, 1 dike and 3 squares, forming a $3.7 \mathrm{~km}$ lakeside landscape belt. There were 118 kinds of main plant species in the wetland park, belonging to 54 families and 97 genera, most of them were green seedlings planted artificially in the later stage $\mathrm{Yu}$, 2009). Jinlong lake wetland park was completed and opened to the public in February 2010.

\section{The research methods}

\subsection{Sample selection and acquisition}

Some studies had shown that the use of photos as a medium for landscape aesthetic evaluation was not significantly different from on-site evaluation, but at the same time, necessary restrictions on sample collection were required (Tripathi \& Gaur, 2006). Therefore, the photos taken at the scene were taken as the aesthetic evaluation samples of plant landscape in this study. Considering the different characteristics of plant landscape construction and the comprehensiveness of sampling in different scenic spots, the plant landscape of each scenic spot in Jinlong lake wetland park was photographed. The photos were taken in the morning in May 20, 2019 and the weather was fine. The camera used for taking photos was Samsung WB650, the shooting height was about $1.6 \mathrm{~m}$, and no flash was used to ensure the consistency of photo shooting technical specifications, 305 photos were taken in total. 35 photos that were the most representative and could fully reflect the plant landscape information were selected from the 305 photos, which were 8 waterfront plant landscapes, 8 plant landscapes on both sides of the roads, 7 plant landscapes beside buildings and surroundings, and 12 typical plant communities. The selected 35 photos were disordered and made into slides as evaluation objects.

\subsection{Evaluators}

Previous studies had shown that: different evaluation groups had obvious consistency in aesthetic attitude, and the perception of relevant experts and professional students was better than that of the general public (Song et al., 2006; Zhang et al., 2012). It was easier for students to be organized than for park visitors. And students of landscape architecture have a good perception of plant landscape aesthetics. Therefore, 63 landscape architecture students (Sophomore) in Xuzhou University of Technology were selected to evaluate the plant landscape aesthetics of Jinlong lake wetland park. Some printed evaluation forms were send to each evaluator and a detailed description of the evaluation requirements was given.

\subsection{Scenic beauty estimation (SBE) method}

Evaluation work was carried out in the classroom. The slide was show once, with an interval of 2 s between each photo, so that the evaluators were familiar with the objects to be evaluated. At the beginning of the second screening, the evaluators evaluated each landscape photo in turn with an interval of 10 s, the evaluation grade was divided into "five levels", namely "excellent", "good", "medium", "general" and "poor", and the corresponding scores were " $8-10,6-8,4-6,2-4,0-2$ " respectively. At the last screening, the evaluators corrected the scores, with an interval of $5 \mathrm{~s}$ for each photo. After checking, there were 4 invalid forms, and 59 valid evaluation forms were obtained. 
In view of the fact that the evaluation results were affected by the characteristics of the landscape sample itself and the individual differences of the evaluators, it was necessary to standardize the original scores to obtain the scenic beauty value of each photo. The formula were:

$$
\begin{aligned}
& Z_{i j}=\left(R_{i j}-\overline{R_{j}}\right) / S_{j} ; \\
& S B E_{i}=\sum Z_{i j} / N_{i} .
\end{aligned}
$$

In formula (1), (2), $Z_{i j}$ was the standardized value of the evaluator $j$ for the landscape photo $i, R_{i j}$ was the original evaluation value of the evaluator $j$ for the landscape photo $i, \overline{R_{j}}$ was the mean value of the original value of all landscape photos evaluated by the evaluator $j$. was the standard deviation of the original value of all landscape photos evaluated by the evaluator $j, S B E_{i}$ was the final standardized scenic beauty evaluation value of the landscape photo $i$.

\subsection{Principal factor analysis method}

According to the characteristics of the plant landscape, and refer to related documents (Zhang \& Wang, 2017; Xie et al., 2016; Tan \& Peng, 2020) and the actual situation of the plant landscape in Jinlong lake wetland park. Plant species (x1), plant layer (x2), plant color (x3), plant space $(\mathrm{x} 4)$, plant posture $(\mathrm{x} 5)$, plant growth $(\mathrm{x} 6)$, and plant artistic conception (x7) were selected as the evaluation elements of plant landscape aesthetics in Jinlong lake wetland park, and the evaluation criteria were established (Table 1). 63 landscape architecture students (Sophomore) scored the plant landscape elements of each photo in classroom and each photo took 60-80 seconds. The effective evaluation table has 55 points. The sample scores were averaged as the quantitative values of plant landscape elements.

SPSS was used to conduct principal factor analysis of the average score of plant landscape elements in 35 photos, which was divided into the following steps: (1) Correlation analysis and KMO and Bartlett tests were conducted to determine whether factor analysis was suitable. (2) According to the eigenvalues and cumulative variance of the principal factors, the number of the principal factors was determined and the characteristics of the principal factors were analyzed. (3) The score value of each principal factor was calculated according to the feature vector, and the comprehensive evaluation value was calculated according to the normalized weight of the contribution rate of each principal factor.

\section{Result analysis}

\subsection{SBE analysis of plant landscape samples}

It could be seen from Table 2 that among the 35 plant landscape samples, the highest score was No. 17 (Figure 1), and the SBE value was 1.2174. No. 17 was the plant landscape on both sides of a road, which was composed of Cercis chinensis and Ophiogon japonicus. Cercis chinensis was densely planted on both sides of the landscape path, and the crown was connected to form an arched upper covering landscape of the road. The second highest SBE value was the typical plant community with No. 9 (Figure 1). Photo No. 9 took the large evergreen plant Ligustrum compactum as the background, and four Acer palmatum 'Atropurpureum were planted in front, with yellow Petunia hybrida (Vilm) and pink Bellis perennis as the ground cover, forming a plant landscape with distinct layers, rich posture and bright colors.

The photo with the lowest score was No. 23 (typical plant community), followed by No. 6 (waterfront plant landscape) (Table 2, Figure 2). In photo No. 23, the plants were arranged in the forms of Celtis bungeana, Ligustrum compactum, Koelreuteria paniculata + Cercis chinensis + Buxus megistophylla + Ophiogon japonicus, Humulus scandens. There were many layers of plants in No. 23, and the canopy density was not high, some big trees had bent trunk and the ground cover was disorderly. In photo No. 6, plants were composed of Metasequoia glyptostroboides + Photinia serrulata, Jasminum nudiflorum in

Table 1. Evaluation criteria of landscape elements

\begin{tabular}{|c|l|l|l|l|l|l|}
\hline \multirow{2}{*}{ Number } & \multirow{2}{*}{$\begin{array}{c}\text { Landscape } \\
\text { elements }\end{array}$} & \multicolumn{1}{|c|}{$0-2$} & \multicolumn{1}{|c|}{$2-4$} & \multicolumn{1}{c|}{$4-6$} & \multicolumn{1}{|c|}{$6-8$} & \multicolumn{1}{c|}{$8-10$} \\
\cline { 3 - 7 } & & \multicolumn{1}{|c|}{$\begin{array}{c}2 \text { species and } \\
\text { below }\end{array}$} & $3-4$ species & $5-6$ species & $7-8$ species & $9-10$ species \\
\hline $\mathrm{X}_{1}$ & Plant species & 1 layer & 2 layers & 3 layers & 4 layers & 5 layers and above \\
\hline $\mathrm{X}_{2}$ & Plant layer & 2 colors and below & 3 colors & 4 colors & 5 colors & 6 colors and above \\
\hline $\mathrm{X}_{3}$ & Plant color & $\begin{array}{l}\text { Canopy density } \\
20 \% \text { and below }\end{array}$ & $\begin{array}{l}\text { Canopy density } \\
20 \%-40 \%\end{array}$ & $\begin{array}{l}\text { Canopy density } \\
40 \%-60 \%\end{array}$ & $\begin{array}{l}\text { Canopy density } \\
60 \%-80 \%\end{array}$ & $\begin{array}{l}\text { Canopy density } \\
80 \% \text { and above }\end{array}$ \\
\hline $\mathrm{X}_{4}$ & Plant space & 1 plant posture & 2 plant postures & 3 plant postures & 4 plant postures & $\begin{array}{l}5 \text { plant posture } \\
\text { and above }\end{array}$ \\
\hline $\mathrm{X}_{5}$ & Plant posture & Poor growth & Average growth & Medium growth & Good growth & Excellent growth \\
\hline $\mathrm{X}_{6}$ & Plant growth & $\begin{array}{l}\text { Poor artistic } \\
\text { conception }\end{array}$ & $\begin{array}{l}\text { Average artistic } \\
\text { conception }\end{array}$ & $\begin{array}{l}\text { Medium artistic } \\
\text { conception }\end{array}$ & $\begin{array}{l}\text { Good artistic } \\
\text { conception }\end{array}$ & $\begin{array}{l}\text { Excellent artistic } \\
\text { conception }\end{array}$ \\
\hline $\mathrm{X}_{7}$ & $\begin{array}{l}\text { Plant artistic } \\
\text { conception }\end{array}$ & & & & \\
\hline
\end{tabular}




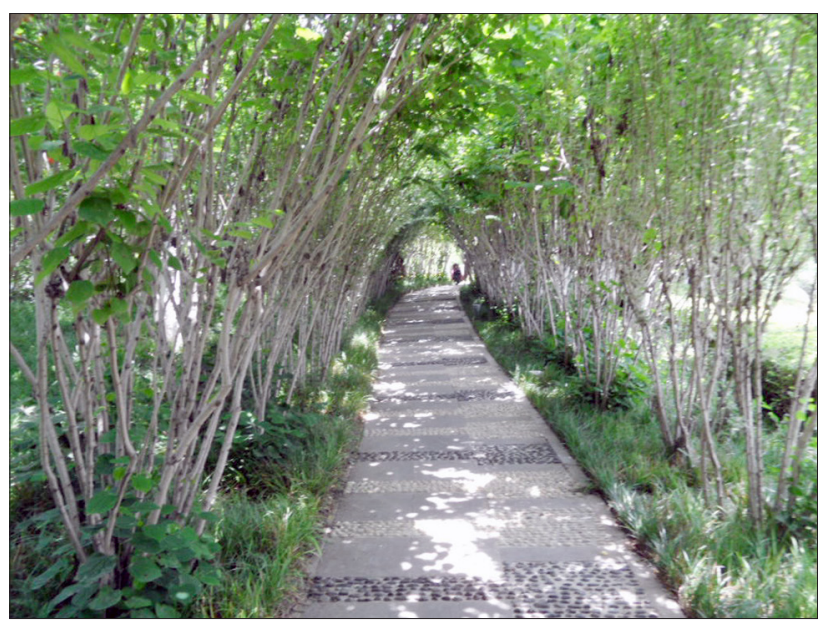

No. 17

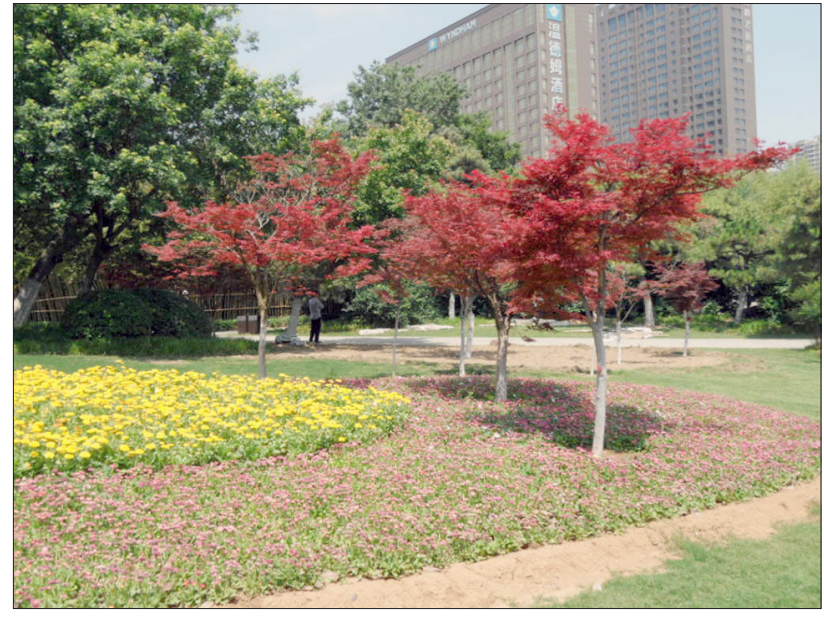

No. 9

Figure 1. Photos with higher SBE value of plant landscape

Table 2. SBE value and ranking of plant landscape in Jinlong lake wetland park

\begin{tabular}{|c|c|c|c|c|c|c|c|c|}
\hline Number & SBE value & $\begin{array}{l}\text { SBE value } \\
\text { ranking }\end{array}$ & Number & SBE value & $\begin{array}{l}\text { SBE value } \\
\text { ranking }\end{array}$ & Number & SBE value & $\begin{array}{c}\text { SBE value } \\
\text { ranking }\end{array}$ \\
\hline 1 & -0.6732 & 33 & 13 & -0.2997 & 28 & 25 & 0.3482 & 13 \\
\hline 2 & 0.3851 & 10 & 14 & 0.3933 & 9 & 26 & 0.3800 & 11 \\
\hline 3 & -0.3242 & 29 & 15 & -0.2430 & 21 & 27 & -0.2967 & 27 \\
\hline 4 & 0.3953 & 8 & 16 & -0.1031 & 18 & 28 & 0.0231 & 15 \\
\hline 5 & -0.0653 & 17 & 17 & 1.2174 & 1 & 29 & -0.6613 & 32 \\
\hline 6 & -0.7092 & 34 & 18 & -0.1706 & 19 & 30 & 0.4470 & 6 \\
\hline 7 & -0.0022 & 16 & 19 & -0.3501 & 30 & 31 & -0.2401 & 20 \\
\hline 8 & -0.2727 & 24 & 20 & 0.5621 & 3 & 32 & 0.5603 & 4 \\
\hline 9 & 0.6677 & 2 & 21 & 0.4627 & 5 & 33 & -0.2831 & 26 \\
\hline 10 & 0.4285 & 7 & 22 & -0.2557 & 23 & 34 & 0.3524 & 12 \\
\hline 11 & -0.2754 & 25 & 23 & -0.8321 & 35 & 35 & -0.6302 & 31 \\
\hline 12 & -0.2445 & 22 & 24 & 0.2911 & 14 & & & \\
\hline
\end{tabular}

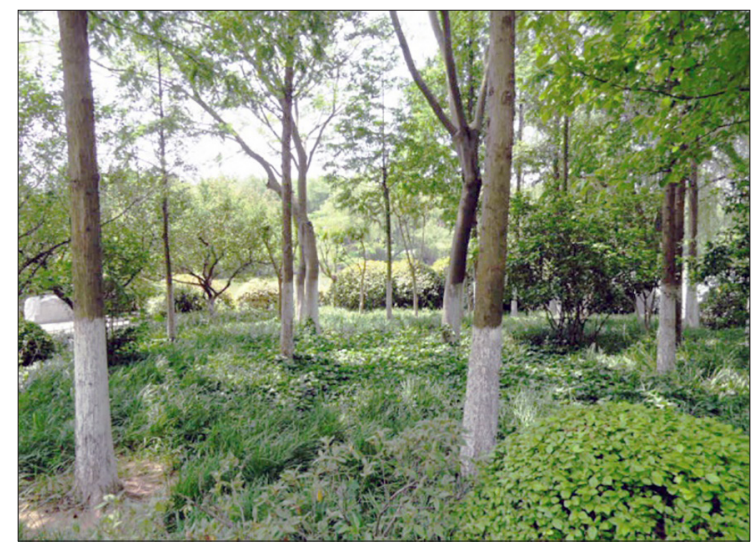

No. 23

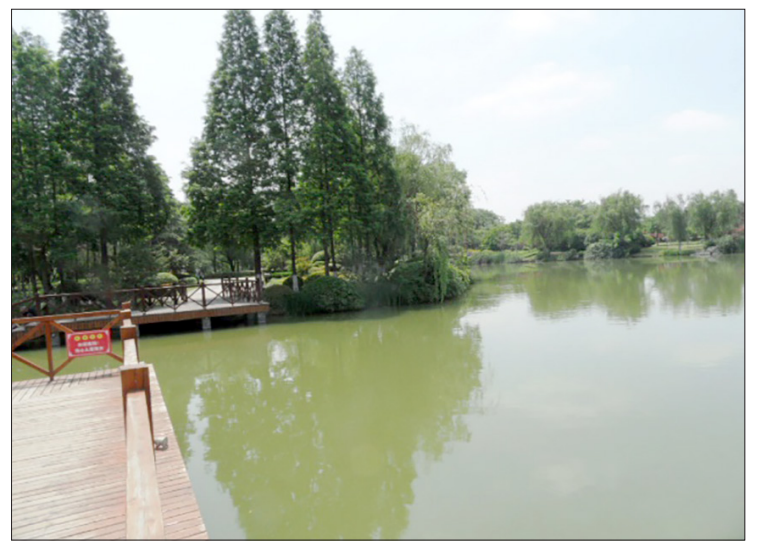

No. 6

Figure 2. Photos with lower SBE value of plant landscape 
the close range, and Salix babylonica + Nerium indicum in the distance, with clear plant arrangement. The low score of photo No. 6 might be due to the fact that the canopy line of distant plants on the opposite side of the river was too straight, the single posture of plants, and the lack of beauty of ups and downs, the warning board on the wooden walkway in the near view and the poor water quality might all affect the evaluation result.

Among the 35 plant landscape samples, 20 had negative SBE values, accounting for $57.14 \%$ of the total number of photos, and 15 had positive SBE values, accounting for $42.86 \%$ of the total, which indicated that the evaluators had a general feeling for the plant landscape of Jinlonghu wetland park. The average value of SBE WAS -0.0005 , but the SBE value fluctuated greatly. The photo No. 17 was the best, and the SBE value was far higher than other photos. The average SBE values of different types of plant landscape samples showed that plant landscape on both sides of the roads $(0.2849)>$ plant landscape beside buildings and surroundings $(0.2224)>$ typical plant community $(-0.1633)>$ waterfront plant landscape $(-0.2224)$.

\subsection{Principal factor analysis of plant landscape samples}

Principal factor analysis was performed on the mean scores of 7 landscape elements of 35 photos. The results showed that there was correlation between the seven landscape elements, the KMO test value was 0.738 , and the sig value of Bartlett spherical test was 0.000 , which was suitable for factor analysis. The cumulative contribution rate of the first three principal factors was $91.449 \%$ (Table 3), which showed that the first three principal factors had reflected $91.449 \%$ of the information of the seven landscape elements. Therefore, the three principal factors were selected as the comprehensive index of plant landscape aesthetic evaluation.

According to the rotation load matrix (Table 4), it could be seen that the principal factor $\mathrm{F}_{1}$ mainly reflected
Table 3. Eigenvalues and variance contribution rate after principal factor rotation

\begin{tabular}{|c|c|c|c|}
\hline $\begin{array}{c}\text { Principal } \\
\text { factor }\end{array}$ & $\begin{array}{c}\text { Eigen- } \\
\text { value }\end{array}$ & $\begin{array}{c}\text { Variance } \\
\text { contribution rate } \\
(\%)\end{array}$ & $\begin{array}{c}\text { Cumulative } \\
\text { variance cont- } \\
\text { ribution rate (\%) }\end{array}$ \\
\hline $\mathrm{F}_{1}$ & 2.861 & 40.869 & 40.869 \\
\hline $\mathrm{F}_{2}$ & 2.186 & 31.228 & 72.097 \\
\hline $\mathrm{F}_{3}$ & 1.355 & 19.352 & 91.449 \\
\hline
\end{tabular}

Table 4. Principal factor rotating load matrix

\begin{tabular}{|l|c|c|c|}
\hline & $\mathrm{F}_{1}$ & $\mathrm{~F}_{2}$ & $\mathrm{~F}_{3}$ \\
\hline Plant species $\left(\mathrm{X}_{1}\right)$ & 0.929 & 0.282 & 0.199 \\
\hline Plant layer $\left(\mathrm{X}_{2}\right)$ & 0.893 & 0.322 & 0.275 \\
\hline Plant posture $\left(\mathrm{X}_{5}\right)$ & 0.726 & 0.361 & 0.527 \\
\hline Plant artistic conception $\left(\mathrm{X}_{7}\right)$ & 0.175 & 0.941 & 0.171 \\
\hline Plant growth $\left(\mathrm{X}_{6}\right)$ & 0.488 & 0.76 & 0.115 \\
\hline Plant color $\left(\mathrm{X}_{3}\right)$ & 0.591 & 0.63 & 0.087 \\
\hline Plant space $\left(\mathrm{X}_{4}\right)$ & 0.236 & 0.121 & 0.955 \\
\hline
\end{tabular}

the information of plant species, plant layer and plant posture, which was named "plant form factor", $F_{2}$ mainly reflected information of plant artistic conception, plant growth and plant color, which was named as "plant aesthetic factor", $F_{3}$ mainly reflected the information of plant space and was named as "plant space factor".

The contribution rate of the three principal factors was normalized, and the weight of each main factor was calculated, so the comprehensive evaluation score:

$$
\mathrm{F}=0.4469 \mathrm{~F}_{1}+0.3415 \mathrm{~F}_{2}+0.2116 \mathrm{~F}_{3} .
$$

The scores of the three principal factors were obtained by multiplying the principal factor score coefficient by the average value of each factor. The comprehensive evaluation value of principal factor of 35 plant landscape samples was shown in Table 5. It could be seen that the

Table 5. Principal factor comprehensive evaluation value and ranking of plant landscape in Jinlong lake wetland park

\begin{tabular}{|c|c|c|c|c|c|c|c|c|}
\hline $\begin{array}{c}\text { Num- } \\
\text { ber }\end{array}$ & $\begin{array}{l}\text { Comprehensive } \\
\text { evaluation value }\end{array}$ & $\begin{array}{c}\text { Comprehensive } \\
\text { evaluation value } \\
\text { ranking }\end{array}$ & $\begin{array}{c}\text { Num- } \\
\text { ber }\end{array}$ & $\begin{array}{l}\text { Comprehensive } \\
\text { evaluation value }\end{array}$ & $\begin{array}{c}\text { Comprehensive } \\
\text { evaluation value } \\
\text { ranking }\end{array}$ & $\begin{array}{c}\text { Num- } \\
\text { ber }\end{array}$ & $\begin{array}{l}\text { Comprehensive } \\
\text { evaluation value }\end{array}$ & $\begin{array}{c}\text { Comprehensive } \\
\text { evaluation value } \\
\text { ranking }\end{array}$ \\
\hline 1 & 3.1539 & 21 & 13 & 3.6943 & 7 & 25 & 3.7318 & 6 \\
\hline 2 & 4.0842 & 2 & 14 & 2.4926 & 33 & 26 & 3.1108 & 22 \\
\hline 3 & 3.5091 & 13 & 15 & 3.3483 & 16 & 27 & 3.5638 & 11 \\
\hline 4 & 4.1589 & 1 & 16 & 2.4825 & 34 & 28 & 3.5524 & 12 \\
\hline 5 & 2.7311 & 31 & 17 & 3.0903 & 23 & 29 & 2.4789 & 35 \\
\hline 6 & 2.5593 & 32 & 18 & 3.3107 & 17 & 30 & 3.7407 & 5 \\
\hline 7 & 3.4635 & 14 & 19 & 2.9876 & 26 & 31 & 3.2761 & 18 \\
\hline 8 & 2.9226 & 28 & 20 & 2.8715 & 29 & 32 & 3.6839 & 8 \\
\hline 9 & 4.0174 & 3 & 21 & 3.5917 & 9 & 33 & 3.2016 & 20 \\
\hline 10 & 3.2610 & 19 & 22 & 2.8715 & 30 & 34 & 3.9644 & 4 \\
\hline 11 & 3.5735 & 10 & 23 & 3.0527 & 24 & 35 & 2.9437 & 27 \\
\hline 12 & 3.0162 & 25 & 24 & 3.3491 & 15 & & & \\
\hline
\end{tabular}


highest score was No. 4 that was a typical plant community landscape (Figure 3), and the comprehensive evaluation value of principal factor was 4.1589 . The plant landscape in photo No. 4 was composed of Ligustrum compactum, Bischofia polycarpa + Buxus megistophylla, Loropetalum chinense + Coreopsis drummondii, Trifolium repens + perennial ryegrass. The plant landscape in photo 4 had a large tree as the background, and the golden Coreopsis drummondi in the front was brightly colored and layered. The second score was photo No. 2 (the plant landscapes on both sides of a road) (Figure 3), which was composed of Robinia pseudoacacia, Metasequoia glyptostroboides, Salix babylonica + Prunus cerasifera, Malus halliana Koehne + Fatsia japonica (Thunb.) Decne. et Planch, Mahonia fortune (Lindl.) + perennial ryegrass. There was rich plant species, diverse spaces, distinct levels and rich posture in photo No. 2. The two photos with lower scores in the comprehensive evaluation of the principal factors were the waterfront plant landscapes of No. 29 and No. 16 (Table 5, Figure 4). In these two photos No. 29 and No. 16, the aquatic plants were Acorus calamus (L.) and Nymphaea tetragona Georgi, but these Nymphaea tetragona Georgi did not form a good

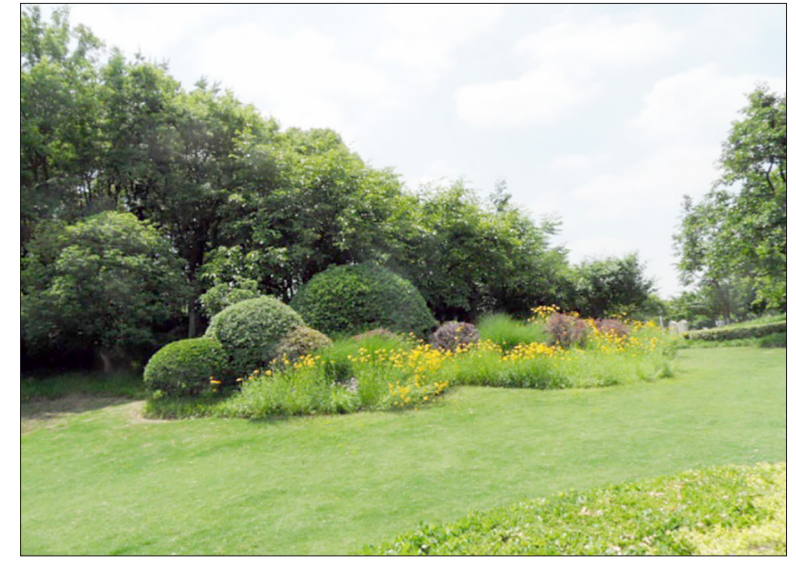

No. 4 water landscape. The plants on the shore mainly included Salix babylonica nearby and Sabina chinensis (L.) Ant. in the distance. The low comprehensive score was mainly due to the low score of the principal factor "plant form factor" in photos No. 29 and No. 16.

Among the 35 plant landscape samples, the comprehensive evaluation scores of principal factors ranged from 2.4789 to 4.1589 , and the scores fluctuated little (Table 5). According to the average value of principal factor comprehensive evaluation value of different types of plant landscape samples, it could be seen that plant landscape on both sides of the roads (3.4177) $>$ typical plant community (3.3275) > plant landscape beside buildings and surroundings (3.2567) > waterfront plant landscapes (3.0966).

\subsection{Comparative analysis of two evaluation methods}

By SBE and principal factor analysis of 35 plant landscape samples, it could be seen that both evaluation had a certain consistency. The average value of plant landscape on both sides of the roads was the first, while the average value of

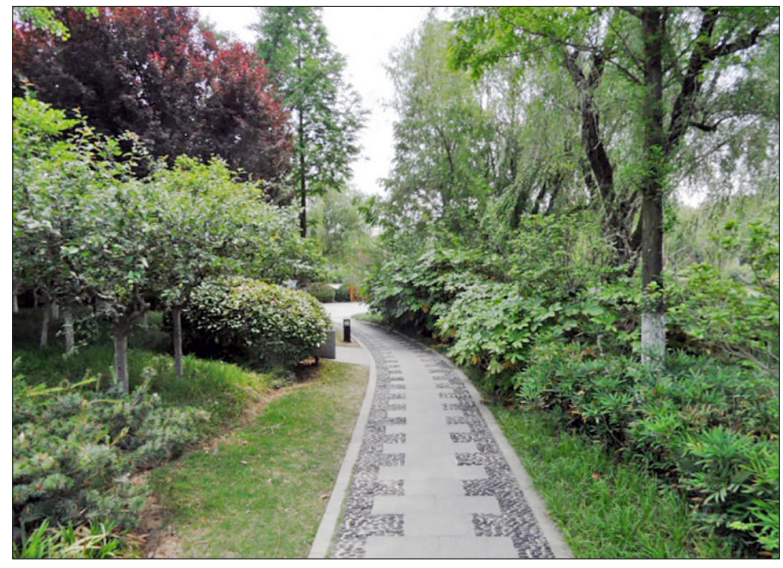

No. 2

Figure 3. Photos with higher principal factor comprehensive evaluation value of plant landscape

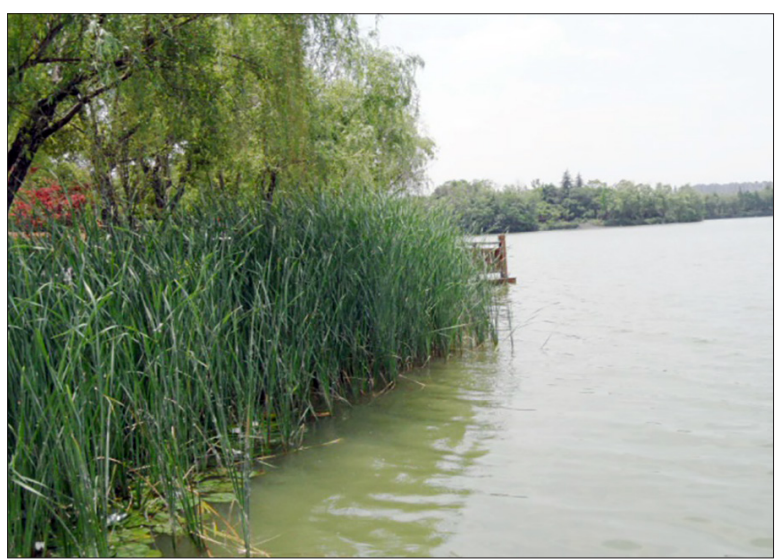

No. 29

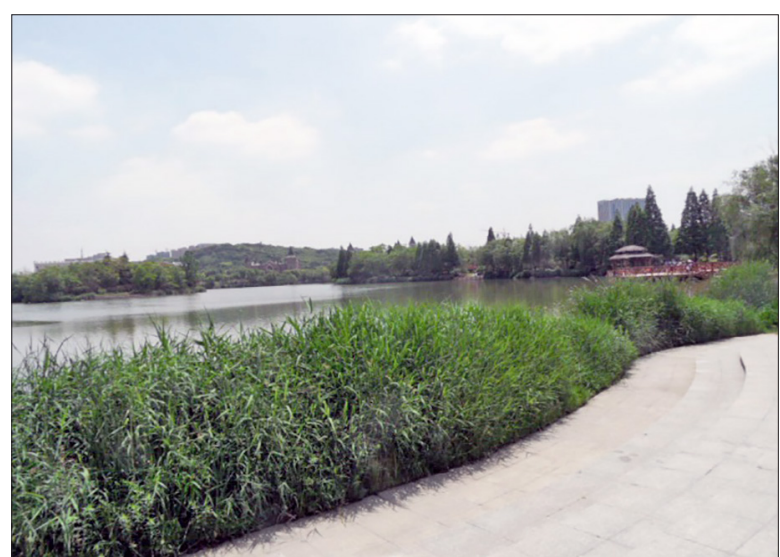

No. 16

Figure 4. Photos with lower principal factor comprehensive evaluation value of plant landscape 
waterfront plant landscape was the lowest, which indicated that the evaluators were satisfied with the plant landscape on both sides of the roads, but the satisfaction degree of waterfront plant landscapes was low. Through pearson correlation analysis of SBE value and principal factor comprehensive evaluation value, it could be seen that their correlation coefficient was $0.403(P=0.016<0.05)$, which was significantly correlated at the level of 0.05 . There were 6 same photos in the top 10, and photo No. 9 in the top 5 according the two evaluation methods.

But there were significant differences between the two evaluation methods. In particular, there was a big difference between the two evaluation results of the photo No. 17, the SBE value was the first, and it was much higher than other landscape photos, but in the comprehensive evaluation of the principal factor, the score was ranked the $23 \mathrm{rd}$. This was mainly because in the principal factor evaluation of photo No. 17, although the main factor "plant aesthetic factor" ranked first, but the principal factor "plant form factor" score was the last one, which led to the unsatisfactory comprehensive evaluation results.

In order to explore the factors affecting SBE value, linear regression analysis was conducted between SBE value and seven landscape elements of principal factor analysis. When $\mathrm{R}^{2}=0.757$, SBE value had significant linear correlation with plant artistic conception and plant color (significance was $0.00<0.01$ ), and other landscape elements were eliminated. The regression equation of SBE value and related factors was: $\mathrm{SBE}=-2.519+0.711 \mathrm{X}_{7}$ (plant artistic conception) $+0.243 \mathrm{X}_{3}$ (plant color), the plant artistic conception and plant color were positively correlated with the SBE value of plant landscape. This indicated that the evaluators may pay more attention to the subjective feeling and visual impact in the scenic beauty evaluation (SBE) in a short period of time, which was mainly affected by the "plant aesthetic factor", while ignoring the "plant form factor" and "plant space factor". The principal factor evaluation method extracted more than $90 \%$ of the information of the 7 landscape factors, especially the "plant form factor", which contributed a lot in the evaluation, which was the main reason for the difference in the results of the two evaluation methods.

\section{Conclusions and discussion}

Plant landscape design was the combination of science and art. In this study, principal factor analysis and SBE were used to evaluate the plant landscape aesthetics of Jinlong lake wetland park in Xuzhou city, and the inherent rules and applicable indicators of plant landscape aesthetic construction were obtained, which was conducive to the study of plant landscape construction. The evaluation results of the two methods had certain consistency and obvious differences. The principal factor analysis method carried on the comprehensive evaluation from seven aspects which were closely related to the plant landscape aesthetics, the subjective and objective factors were combined, and the information contained in the factors was more comprehensive, and it could be analyzed according to different principal factors to find out the problems existing in the plant landscape design and the elements to be considered, so the principal factor analysis was suitable for the overall planning and design of plant landscape. The SBE method was mainly used to evaluate the "plant aesthetic factor", it was highly subjective, but it was easy to form a visual focus, so SBE was suitable for consideration in detailed plant landscape design. The photos No. 17 and No. 9 of the top two SBE values in Jinlong lake wetland park were a good example.

The overall evaluation of plant landscapes on both sides roads in Jinlong lake wetland park was good, with abundant plants, distinct layers and a characteristic Cercis chinensis path. The waterfront plant landscape was less satisfied. As a wetland park, there were less wetland plant species, mainly Phragmites australis, Acorus calamus, Metasequoia glyptostroboides and Salix babylonica, lack of aquatic flowering plants on the shore, heavy artificial traces and lack of natural ecological charm. Therefore, it was necessary to increase the variety of aquatic plants, especially native plants, to reduce the impact of alien plant invasion on the stability of wetland parks (Mitsch \& Hernandez, 2013). The score of typical plant communitiese was higher than that of plant landscapes beside buildings and surroundings in the principal factor comprehensive evaluation, which was mainly because the plants characteristics were more considered. While the SBE method took more consideration of the combination of plants, rocks, buildings and other landscape elements, which was consistent with the previous studies (Weng et al., 2009). The plant landscape transformation of Jinlong lake wetland park mainly focused on waterfront plants, and it was necessary to increase plant species, especially flowering plants, such as Lythrum salicaria, Thalia dealbata Fraser, Nymphaea $L$. and Nelumbo SP. In addition, the construction of natural revetment and the use of plants with different postures to create rich canopy lines were also important means to enhance the aesthetic value of waterfront plant landscape.

\section{Acknowledgements}

This work was financially supported by Science and Technology Planning Projects of Xuzhou City (KC15SM036), Key Cultivation Projects of Xuzhou University of Technology (XKY2017122) and Natural Science Foundation of Jiangsu Province (BK20161164).

\section{References}

An, J., Liu, N. N., Yang, R.H., \& Zhao, J. H. (2014). Scenic beauty evaluation for summer-plant landscape of Huaxi Wetland Park in Guiyang City. Ecological Economy, 30(10), 194-199.

Chen, C., Liu, X. A., \& Yan, L. L. (2018). Evaluation of ecosystem service value of Nanhe National Wetland Park in Sichuan. Wetland Science, 16(2), 238-243.

Chen, Y., Sun, B., \& Liao, S. B. (2014). Scenic beauty estimation of in-forest landscapes in Shenzhen urban forests. Scientia Silvae Sinicae, 50(8), 39-44. 
Duan, M. J., Wang, Y. R., Xie, J. F., \& Guo, J. (2018). Scenic beauty estimation of Beijing urban park plant landscape by scenic beauty estimation method. Science Technology and Engineering, 18(26), 45-52.

Fan, X. C., Dai, C. F., Lu, X. X., \& Fan, Y. W. (2018). Succession and driving factors of phytoplankton functional groups in Jinhewan urban wetland. Acta Ecologica Sinica, 38(16), 5726-5738.

Gobster, P. H., Nassauer, J. I., Daniel, T. C., \& Fry, G. (2007). The shared landscape: What does aesthetics have to do with ecology. Landscape Ecology, 22, 959-972. https://doi.org/10.1007/s10980-007-9110-x

Ji, Y. T., Li, Y. N., \& Chen, J. R. (2016). Evaluation on waterfront plant landscape of urban-park in Chengdu. Journal of Northwest Forestry University, 31(3), 291-297.

Kerebel, A., Gélinas, N., Déry, S., Voigt, B., \& Munson, A. (2019). Landscape aesthetic modelling using Bayesian networks: Conceptual framework and participatory indicator weighting. Landscape and Urban Planning, 185, 258-271. https://doi.org/10.1016/j.landurbplan.2019.02.001

Li, F., Sun, R. H., \& Chen, L. D. (2012). Recreational attraction of urban park wetlands in Beijing. Chinese Journal of Applied Ecology, 23(8), 2093-2099.

Li, M. Y., Wang, H., \& Zhang, M. F. (2015). Study on the optimization of ecological adaptability zoning of Wetland Park based on landscape security pattern. Journal of Southwest Forestry University, 35(5), 52-57.

Lin, J. T., Hu, Y., \& Cao, L. (2013). Planning and design for urban wetland parks based on local characteristics. China Garden, 11, 104-108.

Mitsch, W. J., \& Hernandez, M. E. (2013). Landscape and climate change threats to wetlands of North and Central America. Aquatic Sciences, 75, 133-149. https://doi.org/10.1007/s00027-012-0262-7

Song, L., He, X. Y., \& Xu, W. D. (2006). The measurement of the beauty of urban forest landscape. Chinese Journal of Ecology, 25(6), 621-624.

Tan, X., \& Peng, Y. L. (2019). Ecotourism environment capacity of wetland park in Xuzhou City. Ekoloji, 28(107), 3505-3513.

Tan, X. H., \& Chen, L. Z. (2017). Landscape pattern of urban wetland park based on functional division. Guizhou Agricultural Sciences, 45(11), 114-117.

Tan, X. H., \& Peng, Y. L. (2020). Scenic beauty evaluation of plant landscape in Yunlong Lake wetland park of Xuzhou City, China. Arabian Journal of Geosciences, 13(15), 701. https://doi.org/10.1007/s12517-020-05626-X

Tan, X. H., Peng, Y. L., Huang, S. H., \& Liu, P. (2020). Landscape pattern and ecotourism carrying capacity of Pan'an Lake wetland park in Xuzhou City, China. Desalination and Water Treatment, 188, 288-296.

https://doi.org/10.5004/dwt.2020.25281
Tripathi, B. N., \& Gaur, J. P. (2006). Physiological behavior of Scenedesmus sp. during exposure to elevated levels of $\mathrm{Cu}$ and $\mathrm{Zn}$ and after withdrawal of metal stress. Protoplasma, 229(1), 1-9. https://doi.org/10.1007/s00709-006-0196-9

Valdez, J. W., Hartig, F., Fennel, S., \& Poschlod, P. (2019). The recruitment niche predicts plant community assembly across a hydrological gradient along plowed and undisturbed transects in a former agricultural wetland. Frontiers in Plant Science, 10, 1-9. https://doi.org/10.3389/fpls.2019.00088

Wang, H., Zhang, Y., \& Shen, T. C. (2015). Study on Wetland park planning based on ecological suitability evaluation and landscape pattern analysis. Ecological Economy, 31(12), 185-190.

Weng, S. F., Ke, F., \& Li, C. M. (2009). Study on plant landscape unit of Guangzhou park by AHP and SBE. Chinese Landscape Architecture, 6, 78-81.

Xie, S. S., Wang, D. M., Li, X., \& Kong, D. Z. (2016) Scenic beauty evaluation of plant communities of Zijingshan park in Zhengzhou. Journal of West China Forestry Science, 45(2), 146-152.

$\mathrm{Xu}, \mathrm{L}$., Ou, J., \& Chen, R. J. (2017). Plant aesthetic evaluation of Aha Lake National Wetland Park based on SBE Method. Journal of Mountain Agriculture and Biology, 36(3), 15-22.

Xu, M. F. Zhu, Y. L., \& Cai, P. (2013). Problems and Countermeasures of urban wetland park construction in China. South China Agriculture, 7(5), 24-27.

Yao, X. J., \& Wu, C. H. (2017). The scenic beauty features and the ascension paths of beautiful rural landscape in the north of Anhui Province. Journal of Anhui Agricultural University, 44(6), 1032-1037.

Yu, L. H. (2009). On the shaping of space landscape of Golden Dragon Lake of Xuzhou. Chinese Landscape Architecture, 5, 58-60.

Zhang, J. G., \& Wang, Z. (2017). Research on scenic beauty estimation (SBE) of landscapes of Xiazhu Lake National Wetland Park based on SBE method. Journal of Zhejiang A \& F University, 34(1), 145-151.

Zhang, K. X., Ling, H. R., \& Da, L. J. (2012). Optimization strategies and an aesthetic evaluation of typical plant communities in the Shanghai Green Belt. Acta Ecologica Sinica, 32(17), 5521-5531. https://doi.org/10.5846/stxb201112071874

Zhang, Q. H., Zhao, J., Zhu, J. (2013). Research status of urban wetland park in China. Wetland Science, 11(1), 129-135.

Zhou, C. L., Zhang, Q. X., Sun, Y. K. (2006). Scenic beauty estimation of residential quarter green area. Chinese Landscape Architecture, 4, 62-67.

Zhou, N. X., Huang, Z. F., Jiang, M. P., \& Liang, Y. Y. (2012). The relationship between the aesthetic quality and the landscape pattern index of Lushan forest landscape. Geographical Research, 31(7), 1224-1232. 